\title{
Introducción a la mentoría en investigación y principales marcos teóricos para comprender y explicar sus procesos
}

\author{
Introduction to mentoring in research and main theoretical frameworks to understand and explain their processes
}

Sergio Terrasa ${ }^{a, b, c}$, Carolina Roni ${ }^{d}$, Natalia Rosli ${ }^{d}$, Eduardo Durante ${ }^{a, d}$

\begin{abstract}
Resumen
En este artículo, los autores explican el origen y el uso actual del término mentoría y describen algunos marcos teóricos que ayudan a comprender los procesos y la repercusión de la mentoría en investigación: el del aprendizaje cognitivo, el del aprendizaje no formal, el del aprendizaje sociocultural en el contexto de comunidades de práctica, el de la mentoría como una forma de acumulación de capital social, el de la mentoría como un medio para el desarrollo de la identidad como científico, y las miradas desde la teoría social y cognitiva de la carrera.

Abstract

In this article, the authors explain the origin and the current use of the term mentoring and describe some theoretical frameworks that help to understand the processes and impact of mentoring in research: cognitive learning, non-formal learning, sociocultural learning in the context of communities of practice, mentoring as a form of social capital accumulation, mentoring as a way to develope the identity as a scientist, and the views from the social and cognitive theory of the career.
\end{abstract}

Palabras clave: mentoría, investigación, teoría. Keywords: mentoring, research, theory.

Terrasa S, Roni C, Rosli N, Durante E.. Introducción a la mentoría en investigación y principales marcos teóricos para comprender y explicar sus procesos. Evid Actual Pract Ambul. 2019;22(1):e002004.

\section{Orígenes y uso actual del término mentoría}

La "mentoría" o tutoría es una reconocida estrategia de formación de investigadores en el mundo científico en general ${ }^{1}$ y en el de las ciencias de la salud en particular ${ }^{2,3}$.

La palabra mentoría no existe en el diccionario de la Real Academia Española ${ }^{4}$. Sin embargo, como sostienen Palma Álvarez y col. ${ }^{5}$, es muy frecuente recurrir a este término en los ámbitos educativos y académicos y, especialmente, entre la comunidad científica, donde muchas veces se lo usa directamente en su forma inglesa mentoring*.

Al respecto, Provident ${ }^{6}$ afirma que existen antecedentes del concepto desde la antigua Grecia, pero que fue durante la década del setenta del siglo XX, cuando comenzó a usarse como lo hace la comunidad científica en la actualidad.

Pese a ser una palabra conocida, no hay consenso aún respecto de una única definición del término mentoría ${ }^{7}$. Una de las más aceptadas la considera como un proceso en el cual una persona más experimentada ayuda a otra menos experimentada a ejercer prácticas y a participar en los contextos vinculados a su profesión ${ }^{8-10 \dagger}$

Habitualmente, quien ejerce la mentoría es mayor en edad que quien la recibe y ya ha hecho un camino profesional que le ha permitido posicionarse en su profesión.

Este vínculo transcurre en un entorno de trabajo. Se trata de una relación recíproca que va cambiando (dinámica) y que contribuye a un mejor desarrollo profesional de ambos miembros de la díada ${ }^{10-12 \ddagger}$. Como sostiene Chopra ${ }^{10}$, las palabras mentoría y tutoría evocan, por sí mismas, significados vinculados a aspectos intelectuales y emocionales.

A continuación expondremos algunos marcos teóricos que pueden ayudar a comprender los procesos de mentoría en investigación.

\section{Marcos teóricos que ayudan a comprender los proce- sos de mentoría en investigación}

\section{La mentoría en investigación pensada desde el paradigma del aprendizaje cognitivo}

Ghefaili ${ }^{13}$ reserva el concepto de aprendizaje cognitivo al que implica tareas de cierta complejidad y basadas en problemas. Esta resolución involucra procesos cognitivos y metacognitivos ${ }^{14}$, para los que el aprendiz observa el razonamiento del maestro mientras éste externaliza los procesos mentales de diagnóstico y de resolución de los problemas que van surgiendo, lo que en el lenguaje de las ciencias de la educación se denomina modelaje.

También desde el paradigma del aprendizaje cognitivo, Dennen ${ }^{15}$ hace hincapié en el concepto de intersubjetividad, que implica que el aprendiz y el maestro llegan a la sesión de trabajo, cada uno con su propio entendimiento de lo que se va a trabajar, lo que los obliga a encontrar significados comunes para que la actividad resulte exitosa. Trasladando este concepto a la mentoría en investigación, que casi siempre ocurre en un contexto institucional, podemos agregar que si ambos miembros de la díada no tienen un acuerdo previo respecto del objetivo de aprendizaje

\footnotetext{
a Servicio de Medicina Familiar y Comunitaria, Hospital Italiano de Buenos Aires.

b Departamento de Investigación, Hospital Italiano de Buenos Aires.

c Departamento de Salud Pública, Instituto Universitario Hospital Italiano. sergio.terrasa@hospitalitaliano.org.ar

d Departamento de Educación, Instituto Universitario Hospital Italiano.

* Si bien la palabra mentoría no está incluida en el Diccionario de la Real Academia Española, sí figura la palabra "mentor", con un significado que guarda parentesco con usado el ámbito científico, pero más restringido: "consejero o guía".

$\dagger$ El modelo de la persistencia y el logro académico trata de explicar cómo una persona "calza" con su entorno y sobre las teorías sociológicas sobre la integración en instituciones ${ }^{12}$. Este modelo sostiene que los estudiantes ingresan en las carreras de educación superior con ciertos atributos personales, experiencias escolares y backgrounds familiares. Estos tres niveles pueden influenciar el desempeño escolar, la autopercepción de los estudiantes y sus oportunidades de desarrollo profesional. Por ejemplo, los estudiantes socialmente más integrados (la mentoría puede ser de ayuda en este aspecto) poseen más compromiso institucional, y dicho compromiso, a su vez, se asocia a mejores logros académicos.

$\ddagger$ Quien ejerce la mentoría se beneficia de la obtención de personas aliadas para avanzar en sus líneas de investigación, en el desarrollo de círculos de influencia y en el establecimiento de legados respecto de su liderazgo académico.
} 
y/o desarrollo profesional de quien recibe la mentoría, deben realizar una negociación antes de comenzar a trabajar para mejorar la probabilidad de que ambos -y también la institución- queden satisfechos ${ }^{16}$.

Consideramos que también puede ser aplicado a los procesos de mentoría el difundido concepto de zona de desarrollo proximal -propuesto por Vigotzky ${ }^{13,15}$. Este concepto fue definido como la distancia entre el nivel de desarrollo real y el nivel de desarrollo potencial que sucede cuando se resuelve un problema a partir de la guía de un adulto o compañero más capaz. En nuestro caso, implica que quien ejerce la mentoría procure que el profesional mentoreado salga de su zona de comodidad ante desafíos que realmente pueda afrontar y vencer en ese momento de su desarrollo profesional. Para facilitarle este recorrido, el mentor/tutor puede valerse de alguna estructura de sostén o apoyo, conocida en el lenguaje de las ciencias de la educación como andamiaje (en inglés scaffolding), que ayuda a quien está aprendiendo, a que lleve adelante tareas que inicialmente no podría llegar a desarrollar en soledad. Ese andamiaje suele tener una mezcla artesanalmente equilibrada de ingredientes directivos y de respaldo. Un andamiaje bien implementado tiene dos tipos de repercusiones favorables en quien está comenzando su carrera profesional: 1) repercusiones cognitivas, en términos de desarrollo de habilidades y conocimientos; 2) repercusiones emocionales, a través de un aumento de la motivación y la confianza en sí mismo.

Siguiendo el pensamiento de Ghefaili ${ }^{13}$, desde la mirada del aprendizaje cognitivo puede hacerse una analogía entre el andamiaje que ocurre en los equipos de investigación y el modelo de aprendiz-maestro de cualquier oficio que implique el desarrollo de alguna habilidad artesanal*, pero predominando en los equipos de investigación los procesos de aprendizaje cognitivo.

\section{La mentoría como una forma aprendizaje no formal}

Según Morris ${ }^{17}$, se entiende por aprendizaje formal al que cuenta con objetivos y un currículum definido, una agenda estructurada y evaluaciones formales, por ejemplo los programas de licenciaturas, maestrías o doctorados. Como contrapartida, existe el aprendizaje "no formal", que aunque su nombre pudiera implicar una connotación negativa, tiene un rol extremadamente relevante en el desarrollo de cualquier profesional a lo largo de su carrera de investigación. Si bien el aprendizaje "no formal" podría ser subestimado si se lo somete a la misma vara valorativa que el aprendizaje formal, representa la mayor parte de los procesos de aprendizaje basado en el trabajo que realizan los investigadores noveles que se suman a equipos de investigación ya consolidados.

En relación al aprendizaje no formal, Eraut ${ }^{18}$ ha descripto tres tipos básicos de aprendizaje no formal que Morris ${ }^{18}$ considera válidos para aplicar a la conceptualización del aprendizaje basado en el trabajo. Eraut ${ }^{18}$ llama aprendizaje implícito al que ocurre cuando una persona aprende algo sin darse cuenta y sin habérselo propuesto, como lo que sucede cuando un joven profesional aprende la cultura (p. ej. los lenguajes, los valores, las estrategias comunicacionales, etc.) de la comunidad de investigación en la que se está insertando. El mismo autor ${ }^{18}$ denomina aprendizaje deliberado al que sucede cuando existe un claro propósito de aprender y se dedica a dicha tarea cierto tiempo y energía, por ejemplo, cuando en un equipo de trabajo se solicita al investigador junior que revise un tema y de una clase para el resto de sus compañeros ${ }^{\dagger}$. El tercer tipo de aprendizaje de los que ocurren en los entornos de trabajo es el aprendizaje reactivo, nombre que este autor ${ }^{18}$ da al que sucede en forma no planificada y sorpresiva y luego es reforzado a través de alguna mínima estrategia de consolidación $¥$. Esto implica que tanto el aprendiz y como el equipo en el cual este está inserto deben estar preparados para reaccionar ante el potencial evento sorpresivo promotor del aprendizaje. Por lo tanto, para promover el aprendizaje reactivo en el trabajo, el entorno laboral debe tener una cultura que promueva esta reacción de refuerzo del aprendizaje, por ejemplo, que exista la flexibilidad suficiente para agendar una mini clase no planificada durante la cual quien ha revisado ese tema lo comparta con el resto del equipo, que haya instancias de reflexión grupal y/o de supervisión por pares, etc.

\section{La mentoría en investigación como un proceso de aprendizaje sociocultural}

El enfoque sociocultural del aprendizaje ${ }^{13}$ reconoce que el desarrollo y el aprendizaje humano ocurren por interacciones sociales, y que este aprendizaje depende la cultura y del momento histórico en los que ocurre y se transmite.

A través de la socialización se aprenden las formas acumuladas de pensar y de hacer de cada cultura. Por ejemplo, las habilidades cognitivas (pensar) y lingüísticas (comunicarse) se aprenden a lo largo de la vida de los responsables de la crianza, de los pares y de los maestros.

En el caso que nos compete, -los investigadores jóvenes- esta socialización se realiza en las comunidades de práctica ${ }^{16,19-21}$, en las que tienen un rol relevante quienes ejercen mentoría en investigación.

Lo antedicho implica que el aprendizaje se encuentra anclado a un contexto y ante situaciones auténticas de la práctica académica $^{22 \S}$. El aprendizaje ocurre consecutivamente a la actividad y a la participación en un contexto real de trabajo ${ }^{16}$, no pudiéndose separar al conocimiento de la experiencia, ya que el aprendizaje sucede por la interacción con otros mediante la reflexión, la discusión y la negociación de los significados.

Por otro lado, el desarrollo de la identidad de ser parte de una comunidad de práctica -como cualquier comunidad científica, moldea la adquisición de habilidades y de conocimientos En la medida en que los aprendices van ejerciendo acciones que forman parte de las prácticas profesionales y participan en los contextos específicos (científicos, profesionales, académicos, etc.) empiezan a pertenecer a esos grupos sociales y a desarrollar identidades afines. El ejercicio de las acciones en contextos

* En estos casos aprendiz es pagado por su empleador para aprender y para trabajar. El maestro artesano se muestra como modelo, exhibiendo su práctica al aprendiz. El aprendiz va realizando una práctica supervisada con responsabilidades y autonomía progresivamente crecientes, a la vez que va aprendiendo la tarea y la cultura en la que comienza a verse inserto. Si bien este vínculo de aprendizaje-trabajo comienza con una inversión inicial del empleador, con el tiempo, el maestro captura una parte de lo producido por el aprendiz. Por otro lado, algún día el aprendiz puede llegar volverse autónomo y quizás convertirse en "maestro"

$\dagger$ Implica identificar áreas de debilidad en el desempeño y planificar actividades para mejorarlo.

$\ddagger$ Si bien el aprendizaje reactivo está relacionado con el aprendizaje implícito, ya que es reactivo a los estímulos ambientales que el novato va recibiendo, a diferencia de este, que ocurre sin que el aprendiz se dé cuenta de lo que está aprendiendo (ej. internalización de los valores de una comunidad), quien realiza un aprendizaje reactivo tiene registro de lo que está aprendiendo y luego invierte un mínimo de tiempo y energía más allá del necesario para resolver la necesidad que surgió en el entorno de trabajo.

$\S$ Se aprende mejor en situaciones verdaderas que en las simuladas en los diferentes dispositivos didácticos como son las conferencias o incluso los talleres que emulan situaciones reales y suponen una participación activa del alumno.

T En este sentido vale rescatar el concepto de cognición compartida, que implica que el conocimiento está enormemente distribuido en la experiencia de diferentes personas, en saberes de transmisión oral de generación en generación, en revistas científicas y libros, en Internet, en grandes bases de datos, etc. El ensamblaje de dicho conocimiento implica interacción social. 
auténticos es la herramienta de socialización y aprendizaje privilegiada para la configuración de saberes e identidades. Aunque, en un principio, estas actividades son periféricas y con bajo grado de autonomía, siempre son relevantes

\section{La mentoría como una oportunidad de acumulación de capital} social

Las teorías del capital social proponen que las relaciones sociales regulan el acceso a los recursos y a la información (capital social) ${ }^{23}$, lo que determinará la probabilidad que tendrá cada persona de que sus ideas sean escuchadas y/o tenidas en cuenta, con el consiguiente impacto sobre sus oportunidades de avance a lo largo de su carrera como científico. Las teorías del capital social sugieren que quienes ejercen la mentoría ayudan a las personas que la reciben a aprender los valores que predominan en ese momento histórico en sus profesiones y/o sus campos de estudio, por ejemplo, a qué se considera una buena investigación de acuerdo a dichos valores, y a que desarrollen contactos con otras personas y/o grupos que los ayuden a insertarse en alguna comunidad de práctica. Dicho de otra forma, una buena mentoría contribuye a que quien la recibe se inserte en la red de relaciones propias de quienes investigan y, por lo tanto, acumule capital social.

La influencia de la mentoría en el desarrollo de la identidad como científico y la persistencia académica

Varios autores, como Manson ${ }^{24}$, han propuesto modelos para explicar el camino académico de las personas. Por ejemplo, el reconocimiento de uno mismo como un potencial científico y el reconocimiento de dicha potencialidad por parte de otras personas (por ejemplo, los mentores), ejercería una influencia sobre el desarrollo de la identidad del profesional que se está formando y sobre su comportamiento futuro ${ }^{12}$. Por eso, otro marco teórico relevante para pensar la mentoría en investigación es aquel que considera cómo participa este tipo de vínculo en el desarrollo de la identidad como científico, y su influencia sobre el compromiso con el desarrollo de una carrera académica. Como documentaron Chemers ${ }^{25}$ y col., la mentoría tendría influencia en el desarrollo de la identidad como científico de dos maneras. Una ella es directa, a través del componente socioemocional del proceso de mentoría, mientras que la otra es indirecta y mediada por la percepción de autoeficacia como científico y para el liderazgo y el trabajo en equipo, que a su vez son influenciadas por la calidad de la mentoría recibida Figura 1.

Figura 1. Patrón de correlaciones entre las distintas variables latentes que componen modelo teórico propuesto por Chemers para explicar el desarrollo de la identidad como científico y su influencia sobre el compromiso con la carrera científica. ${ }^{\dagger}$

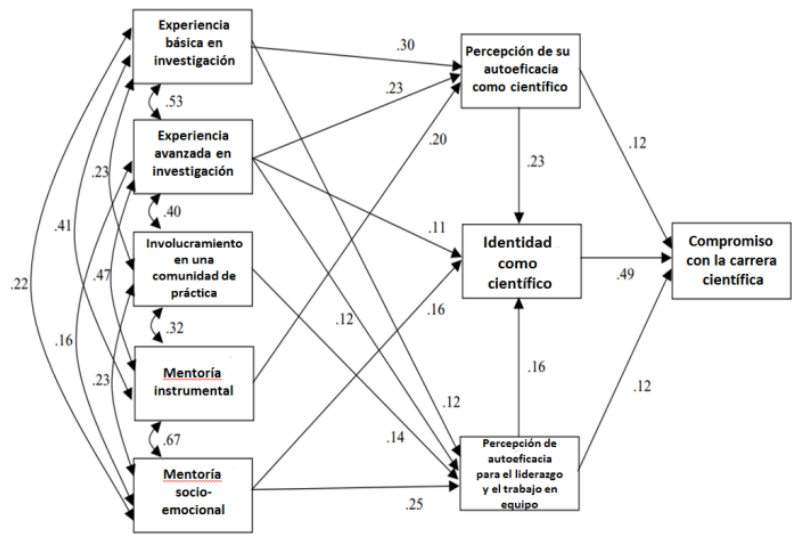

\footnotetext{
${ }^{\dagger}$ Modificado de: Chemers M y col. The Role of Efficacy and Identity in Science Career Commitment Among Underrepresented Minority Students. J Soc Issues. 2011;67(3):469-491. Model Fit: X2 (12): 54.42, $<0.001$. CFI = 0.96, IFI = 0.96, GFI = 0.97, NNFI = 0.87, RMR =0.03, RMSEA =0.10 (0.08 a $0.13) . A I C=30.42$
}

\section{La mentoría entendida desde la teoría social y cognitiva de la carrera}

Sobre la base de los trabajos previamente publicados por Bandura $^{26}$, la teoría social y cognitiva de la carrera propuesta por Lent y col. ${ }^{27}$ articula los mecanismos que subyacen a la motivación individual, al establecimiento de metas, al sendero que cada persona transcurre a lo largo de su carrera profesional y a la persistencia hacia algún objetivo académico.

Esta teoría propone que las personas fortalecen su interés en alguna determinada actividad cuando sienten que pueden volverse competentes para realizarla (percepción de auto-eficacia) y cuando consideran que conducirá a resultados valiosos. Por otro lado, los desenlaces que van ocurriendo (éxitos o fracasos en las distintas instancias) proveen un feedback que fortalece o debilita la percepción de su autoeficacia y la del valor de sus resultados, y conducen a que dichas elecciones sean reforzadas o revisadas.

Más allá de las características individuales de cada persona, esta teoría también sostiene que las elecciones educacionales y profesionales son influenciadas por estímulos contextuales como la mentoría, que pueden actuar potenciando a la persona o bien, volverse una barrera para su desarrollo.

\section{A modo de resumen}

Luego de este recorrido por diversos marcos teóricos podemos concluir transitoriamente que, si bien existen algunas dificultades lingüísticas que complican un poco la traducción del término mentoría, las presiones globalizantes sobre el lenguaje están facilitando que el concepto sea trasladable y comprensible en ámbitos de habla hispana, aunque no exista una definición clara en el Diccio- 
nario de la Real Academia Española.

Podemos concluir además que la mentoría puede ser entendida como un proceso de enseñanza y también como un proceso de aprendizaje sociocultural de fuertes características no formales en el que suceden mecanismos implícitos, deliberados y reactivos. También puede ser de utilidad el paradigma del aprendizaje cognitivo para comprender cómo aprende quien lo hace, espe- cialmente a través del modelaje y del andamiaje.

Por último, una buena mentoría contribuye a que quien la recibe acumule capital social y refuerce su identidad como científico, a través del refuerzo de su percepción de autoeficacia y de potenciar su persistencia en el camino académico.

Recibido el 01/08/2018 y aceptado el 04/03/2019.

\section{Referencias}

1. Roch G. Much ado about mentors. Harv Bus Rev. 2016:57(1):14-20. Available from: http://www.ncbi.nlm.nih.gov/pubmed/10244210.

2. Sambunjak D, Straus SE, Marušić A. Mentoring in Academic Medicine. . JAMA. 2006;296(9):1103-1115. Available from: 10.1001/jama.296.9.1103.

3. Eby LT, Allen TD, Evans SC, Ng T, Dubois D. LT, Allen TD, Evans SC, Ng T, Dubois D. Does Mentoring Matter? A Multidisciplinary Meta-Analysis Comparing Mentored and Non-Mentored Individuals. J Vocat Behav. 2008;27(2):254-267. Available from: 10.1016/j.jvb.2007.04.005.

4. Real Academia Española. Diccionario de La Lengua Española - Edición Del Tricentenario. Madrid: Espasa; 2015.Available from: http://dle.rae.es.

5. Álvarez GP, Tovar AO. La mentoría en investigación; 2011. Available from: https://umshare.miami.edu/web/wda/ethics/PABI_Agendas/colombia/ Modules/Module14705.pdf.

6. Provident IM. Provident IM. Mentoring: A Role to Facilitate Change. . Internet J Allied Heal Sci Pract. 2005;3(2).

7. Wocial LD. The role of mentors in promoting integrity and preventing scientific misconduct in nursing research. J Prof Nurs. $1995 ; 11(5): 276-280$. Available from: 10.1016/S8755-7223(05)80008-0.

8. Abedin Z, Biskup E, Silet K. Deriving Competencies for Mentors of Clinical and Translational Scholars. Clin Transl Sci. 2012;5(3):273-280. Available from: 10.1111/j.1752-8062.2011.00366.x.

9. Allen TD, Eby LT, Poteet ML, Lentz E, Lima L. Career benefits associated with mentoring for protégeé: a meta-analysis. J Appl Psychol. 2004;89(1):127-136. Available from: 10.1037/0021-9010.89.1.127.

10. Chopra V, Edelson DP, Saint S. Mentorship Malpractice. JAMA. 2016;315(14):1453. Available from: doi:10.1001/jama.2015.18884.

11. Sambunjak D, Straus S, Marusic A. A systematic review of qualitative research on the meaning and characteristics of mentoring in academic medicine. J Gen Intern Med. 2010;25(1):72-78. Available from: doi:10.1007/s11606-009-1165-8.

12. Pfund C, Byars-Winston A, Branchaw J, Hurtado S, Eagan K. Defining Attributes and Metrics of Effective Research Mentoring Relationships. AIDS Behav. 2016;20(Suplemento):238-248. Available from: 10.1007/s10461-016-1384-z.

13. Ghefaili A. Cognitive Apprenticeship, Technology, and the Contextualization of Learning Environments. Journal of Educational Computing, Design \& Online learning . 2003;4:1-27.

14. Stalmeijer RE. When I say ... cognitive apprenticeship. Med Educ. 2015;49(4):355-356. Available from: 10.1111/medu.12630.

15. Dennen VP. Cognitive apprenticeship in educational practice: Research on scaffolding, modeling, mentoring, and coaching as instructional strategies. In: Jonassen $\mathrm{H}$, et al., editors. Handbook of research on educational communications and technology. vol. 2004. US: Lawrence Erlbaum Associates Publishers;. p. 813-828.

16. Díaz-Barriga F. Cognición situada y estrategias para el aprendizaje significativo. . Rev electrónica Investig Educ. 2003;5(2):105-117.

17. Morris C. Work-based Learning. In: Swanwick T, Forrest K, O'Brien BC, editors. Understanding Medical Education: Evidence, Theory and Practice. Wiley-Blackwell; 2019. p. 163-178.

18. Eraut M. Non-formal learnin, implicit learning and tacit knowledge in professional work. Br J Educ Psychol. 2000;70(1):113-136. Available from: $10.1348 / 000709900158001$.

19. Byars-Winston A, Gutierrez B, Topp S, Carnes M. Integrating theory and practice to increase scientific workforce diversity: A framework for career development in graduate research training. CBE Life Sci Educ. 2011;10(4):357-367. Available from: 10.1187/cbe.10-12-0145.

20. Lave J. Situating Learning in Communities of Practice. In: Resnick B, Levine JM, Teasley SD, editors. Perspectives on Socially Shared Cognition. Washington, DC, US: American Psychological Association; 1991. p. 63-82. Available from: http://dx.doi.org/10.1037/10096-003.

21. Smith MK. Communities of practice, the encyclopedia of informal education. Encycl informal Educ. 2003;p. 1-8.

22. Zaidi S, Nasir M. Teaching and Learning; 2015.Available from: 10.1007/978-3-319-06850-3.

23. Ramírez-Plascencia J. Tres visiones sobre Capital Social: Bourdieu, Coleman y Putnam. Acta Republicana. 2005;4(4):21-36. Available from: http://148.202.18.157/sitios/publicacionesite/pperiod/republicana/pdf/ActaRep04/articulos21.pdf.

24. Manson SM. Personal journeys, professional paths: persistence in navigating the crossroads of a research career. Am J Public Health. 2009;99(Suppl 1):S-20-S-25. Available from: 10.2105/AJPH.2007.133603.

25. Chemers M, Zurbriggen E, Syed M, Goza B, Bearman S. The Role of Efficacy and Identity in Science Career Commitment Among Underrepresented Minority Students. J Soc Issues. 2011;67(3):469-491. Available from: 10.1111/j.1540-4560.2011.01710.x;https://doi.org/10.1111/j.1540-4560.2011. 01710.x.

26. Bandura A. Social Cognitive Theory of Self-Regulation. Organ Behav Hum Decis Process. 1991;50:248-287. Available from: http://www.uky.edu/ reushe2/BanduraPubs/Bandura1991OBHDP.pdf.

27. Lent R, Brown S, Hackett G. Toward a Unifyng Social Cognitive Theory of Career and Academic Interest, Choice and. Performance J Vocat Behav. 1994;45(1):79-122. Available from: 10.1006/jvbe.1994.1027;https://doi.org/10.1006/jvbe.1994.1027. 